EPJ Web of Conferences 52, 12001 (2013)

DOI: 10.1051/epjconf/20135212001

(C) Owned by the authors, published by EDP Sciences, 2013

\title{
Theory Summary: Very High Energy Cosmic Rays
}

\author{
Subir Sarkar ${ }^{1, a}$ \\ ${ }^{1}$ Rudolf Peierls Centre for Theoretical Physics, University of Oxford, 1 Keble Road, Oxford OX1 3NP, UK
}

\begin{abstract}
This is a summary of ISVHECRI 2012 from a theorist's perspective. A hundred years after their discovery, there is renewed interest in very high energy cosmic rays and their interactions which can provide unique information on new physics well beyond the Standard Model if only we knew how to unambiguously decipher the experimental data. While the observational situation has improved dramatically on the past decade with regard to both improved statistics and better understood systematics, the long standing questions regarding the origin of cosmic rays remain only partially answered, while further questions have been raised by new data. A recent development discussed at this Symposium is the advent of forward physics data from several experiments at the LHC, which have broadly vindicated the air shower simulation Monte Carlos currently in use and reduced their uncertainties further. Nevertheless there is still a major extrapolation required to interpret the highest energy air showers observed which appear to be undergoing a puzzling change in their elemental composition, even casting doubt on whether the much vaunted GZK cutoff has indeed been observed. The situation is further compounded by the apparent disagreement between Auger and Telescope Array data. A crucial diagnostic will be provided by the detection of the accompanying ultra-high energy cosmic neutrinos - two intriguing events have recently been recorded by IceCube.
\end{abstract}

\section{Slides}

The slides of the talk can be found on the website of the symposium ISVHECRI 2012: https://indico.desy.de/conferenceOtherViews.py?view=standard\&confId=4594

\footnotetext{
as.sarkar@physics.ox.ac.uk
} 